$\begin{array}{lll}\begin{array}{l}\text { Submission: 13/Jan/2021; } \\ \text { Camera ready: 13/Jul/2021; }\end{array} & \begin{array}{l}1^{\text {st }} \text { round notif.: 05/May/2021; } \\ \text { Edition review: 22/Jul/2021; }\end{array} & \begin{array}{l}\text { New version: 12/May/2021; } \\ \text { Available online: 06/Aug/2021; } \quad \text { Published: 06/Aug/2021; }\end{array} \\ \end{array}$

\title{
Mediación tecnológica apoyada en la cultura Maker en educación secundaria
}

\author{
Title: Educational technological mediation supported by Maker culture in secondary \\ education
}

\author{
Martín-Santiago \\ Domínguez-González \\ Benemérita Universidad Autónoma \\ de Puebla \\ Facultad de ciencias \\ de la electrónica \\ martin.dominguezg@alumno.buap.mx
}

\author{
Daniel \\ Mocencahua-Mora \\ Benemérita Universidad Autónoma \\ de Puebla \\ Facultad de ciencias \\ de la electrónica \\ daniel.mocencahua@correo.buap.mx
}

\author{
Juan-Manuel \\ González-Calleros \\ Benemérita Universidad Autónoma \\ de Puebla \\ Facultad de ciencias \\ de la computación \\ juanmanuel.gonzalez@correo.buap.mx
}

\section{Resumen}

Muchos han sido los esfuerzos que se han hecho a nivel mundial sobre el empoderamiento tecnológico de los docentes en todos los niveles. No obstante, en nuestro contexto estos esfuerzos no han rendido frutos y no se puede observar un uso, ya no digamos adecuado, de la mediación tecnológica en educación. En este artículo se muestra un análisis cualitativo del fenómeno de la adopción efectiva por el docente, de la mediación tecnológica educativa. Que se apoya en la cultura Maker, ampliamente divulgada y adoptada en muchos países. El diseño metodológico fue investigación -acción con profesores de ciencias naturales y tecnología en educación secundaria, desarrollada a través de la capacitación de los profesores y la acción del docente en el aula. Se destacan hallazgos, como la apropiación de la cultura Maker por los docentes, altas expectativas sobre su acción en el aula, la tutoría entre pares, la actitud colaboradora, el aprendizaje de nuevas cosas, entre otros.

Palabras-Clave: Investigación cualitativa, investigación-acción, construccionismo, andragogía, educación secundaria.

\begin{abstract}
Several efforts have been made to empower teachers at all levels of education with the use of technology for education. However, in our context these efforts have not been produce benefits, as there is no evidence of the use, not mentioning correct use, of technological mediation in education. In this article we show a qualitative analysis of the phenomenon of effective adoption of technological mediation in education. We rely on the Maker culture, widely disseminated, and adopted in many countries. Methodological design was research-action with professors of natural sciences and technology in secondary education, with the training of teachers and the action of the teacher in the classroom. Insights include findings, such as the appropriation of Maker culture by teachers, high expectations about their action in the classroom, peer work, collaborative attitude, learning new things, among others.
\end{abstract}

Keywords: Qualitative research, action research, constructionism, andragogy, secondary education.

Cite as: Domínguez-González, MS., Mocencahua-Mora, D., \& González-Calleros, JM. (2021). Mediación tecnológica apoyada en la cultura Maker en educación secundaria. Revista Brasileira de Informática na Educação, 29, 775-797. DOI: 10.5753/RBIE.2021.29.0.775 


\section{Introducción}

Los resultados del programa PISA de ciencias en México aplicado en el año 2015 son contundentes, se identifica que la principal causa del bajo desempeño de los estudiantes en dicha materia al terminar la secundaria es la forma en que los profesores enseñan éstas, es decir la práctica docente; situación que, a pesar de estos resultados, contrasta con un alto interés de los jóvenes por éstas (OCDE, 2016).

Otro argumento que confirma lo anterior es el que se refiere a la forma de cómo se ha enseñado ciencias en los cursos de educación básica en México. Que es una práctica apegada al dictado y a la memorización, lo que no ha permitido la construcción de conocimiento en los estudiantes según Flores-Camacho (2012). Ambos argumentos enfatizan un problema que ha existido en la enseñanza de dicha materia, debido a la práctica docente en secundaria, que se ha mantenido sin cambios significativos.

En el documento emitido por la Secretaría de Educación Pública (SEP), Los aprendizajes clave para la educación integral para la educación básica, se enuncia para los docentes, que los conceptos de ciencias deben ser asociados con la práctica y la acción, y se deben desarrollar proyectos como estrategia didáctica. En este sentido, se plantea una acción renovadora de la enseñanza de éstas, que demanda a los profesores a modificar de manera significativa su práctica docente (SEP, 2017).

El objetivo de este trabajo es dar a conocer una investigación aplicada de corte cualitativo, bajo un diseño de investigación-acción, de una propuesta de mediación tecnológica educativa apoyada en la cultura Maker, con docentes que imparten materias de ciencias y tecnología en educación secundaria en Puebla, México. Quienes aceptaron participar de manera voluntaria en un proceso de capacitación y acompañamiento, por el interés de realizar un cambio de su práctica docente. Esta investigación se realizó en dos eventos, el primero en el periodo escolar 2017-2018 y el segundo en el 2018-2019.

Este proyecto partió con una propuesta con ciertos conceptos y teorías; y conforme se avanzó en su proceso investigativo se fueron integrando otros, hasta lograr una articulación del marco teórico conceptual que se muestra en la Figura 1.

Con bloques de diferentes colores se identifican la correspondencia a los marcos epistémico, teórico, conceptual y metodológico. Esta articulación inicia en el problema de investigación que parte de los resultados de PISA 2015 aplicado a ciencias en educación secundaria en México, y los argumentos sobre la enseñanza de éstas, en la educación básica en México. Este fue el punto de partida que implicó el desarrollo de una investigación que se percibe desde la actividad profesional del docente, con un diseño metodológico de investigación-acción, donde se involucraron investigador y docentes, como participantes en un proceso de reflexión y acción en pro de la mejora de su práctica docente (Hernández et al., 2010). Que dio lugar al planteamiento de la propuesta de mediación tecnológica para la enseñanza de ciencia y tecnología en educación secundaria apoyada en la cultura Maker (Muñoz, 2016).

El investigador tomó el rol de cooperador, entendiendo la acción de cooperar a hacer algo para que junto a la acción o el esfuerzo de otra u otras personas se consiga un resultado. Para este caso, el aporte del investigador es en el ámbito del conocimiento tecnológico y los argumentos de cambio para promover la mejora de la práctica docente. El profesor tomó el rol de investigador para qué, con el conocimiento de su realidad y experiencia en el campo de trabajo en el aula, desarrolle su propia estrategia de enseñanza tras la búsqueda de la mejora de su práctica docente (Martínez, 2007).

Los participantes se apoyan en la cultura Maker como un agente transformador de aprendizaje, al aprovechar la relación de la tecnología y la sociedad que la caracteriza, al emplear 
el conocimiento compartido que existe en internet y hacer tecnología por él mismo y con otros docentes, lo que resulta en un conocimiento al alcance del docente para generar la propuesta de mediación tecnológica que se desarrolle en el aula (Tesconi, 2015).

Conforme se avanzó en la investigación se integraron otros elementos. Se consideró necesaria la integración de la tecnología en el rol del docente en el aula, por lo que se tomó como referente el modelo TPACK (Technological Pedagogical Content Knowledge), propuesto por Koehler y Mishra citado en Cabero et al., (2017) que menciona tres tipos de conocimiento que el docente debería dominar; el conocimiento del contenido (CK), el pedagógico (PK) y el tecnológico (TK). Refiriéndose como conocimiento tecnológico a las Tecnologías de Información y Comunicación (TIC). En este caso, se decidió extender TK a la tecnología de hardware y usarlo como modelo en su formación del docente.

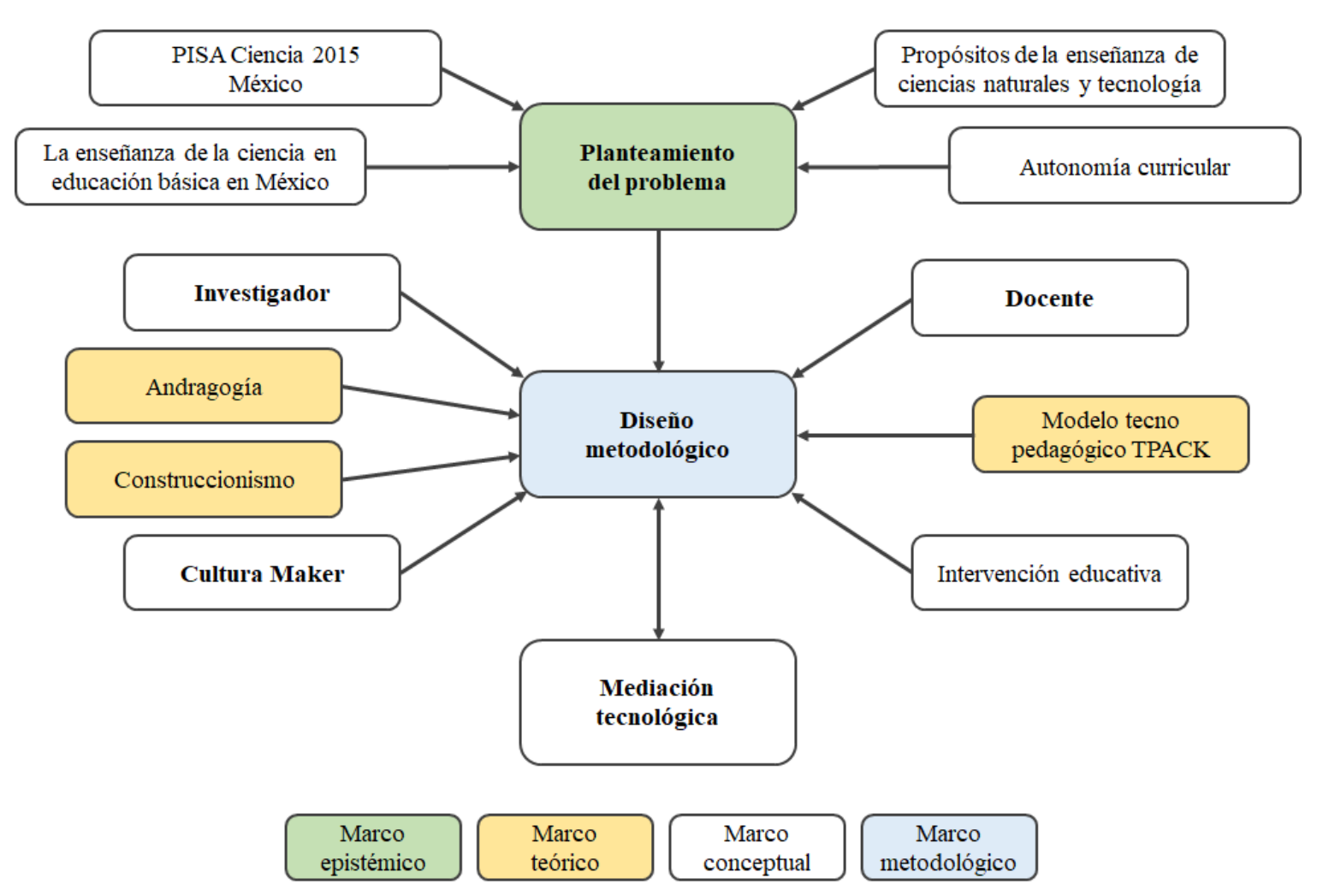

Figura 1. Articulación del marco teórico conceptual.

El docente realiza una intervención educativa en el aula, convencido de desarrollar acciones diferentes en su práctica docente (Pérez, 2017). Esta intervención se justifica por la visión y los propósitos de la enseñanza de las ciencias naturales y tecnología, descritos en los Aprendizajes clave para la educación 2017 en SEP (2017), que indican, en términos generales, ver las ciencias más vinculadas con la tecnología; así como la autonomía curricular, por ser cursos y talleres que se imparten para ampliar la formación académica con nuevos contenidos relevantes, conocimientos necesarios en la región y proyectos de impacto en la comunidad. Esto amplió la aceptación de esta investigación por los docentes, no solo de los que imparten ciencias sino también quienes imparten actividades tecnológicas o curso de autonomía curricular relacionado con ciencias y tecnología.

Los participantes se apoyaron en la aplicabilidad que tiene la cultura Maker en la educación, relacionada con el Construccionismo. Este se integra en la articulación teórico conceptual, en concordancia con argumentos descritos en Tesconi (2015), quien nos habla del gran potencial que 
tiene esta cultura en la educación. El construccionismo, desarrollado por Seymour Papert, nos dice que el aprendizaje se relaciona con la producción de artefactos, los cuales funcionan como elementos activadores del proceso de construcción del conocimiento.

El trabajo cooperativo del investigador con los docentes implicó atender el aprendizaje en el adulto (andragogía) al considerar su conocimiento y percepción previa del tema, para entablar una relación mayormente horizontal donde se facilite la delegación de tareas y poder tomar acuerdos en grupo que ayudaron al compromiso individual y animaran al cambio (Walker y Montero, 2004).

\section{Método}

El primer evento del trabajo de campo se desarrolló durante el ciclo escolar 2017 - 2018, en las fechas descritas de la Tabla 3 a continuación.

Los elementos que corresponden al diseño metodológico de esta investigación se presentan en secuencia: se inicia estableciendo una investigación de corte cualitativo con un diseño metodológico de investigación-acción, el trabajo de campo, la recolección y el análisis de los datos con los cuales se tendrán los resultados de la investigación, que se muestra en la Figura 2 como la articulación metodológica.

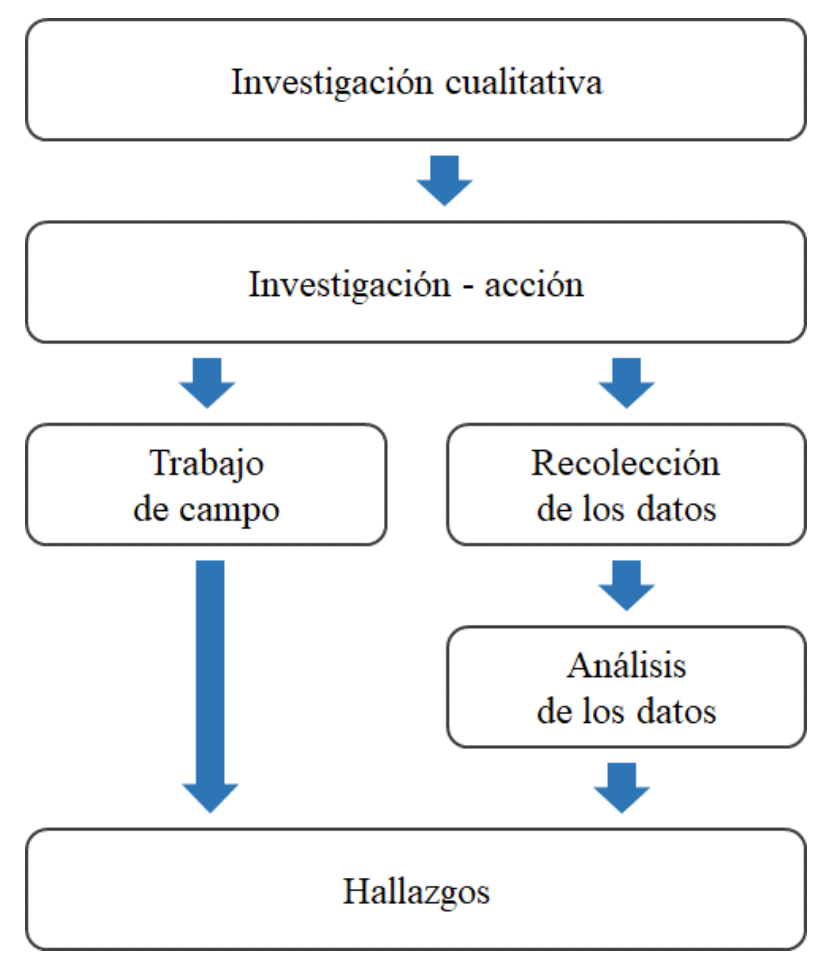

Figura 2. Articulación metodológica.

De acuerdo con Sabariego y Bisquerra (2009), la selección del enfoque y el método de investigación, adecuados al problema depende de la orientación pretendida por el investigador y el objetivo de la investigación. La primera se identifica por desarrollar un proceso de cambio por el docente y conocer lo que ocurre en este proceso. De esta manera, en este trabajo, la selección del método de investigación es considerada dentro de los métodos orientados a solucionar problemas prácticos. En cuanto al objetivo, el estudio se orienta a adoptar la investigación con 
enfoque cualitativo, por la necesidad de lograr el acercamiento con docentes, en el contexto particular que se planteó: educación secundaria.

Derivado del problema de investigación se tiene un escenario en la escuela secundaria en México, con los docentes que enseñan materias de ciencias naturales y tecnología. Como fue mencionado anteriormente se adoptó el modelo TPACK como referente, particularmente para asignar las tareas que desarrollan el investigador y el docente por las capacidades e interacciones que ejecutan, de acuerdo con el contenido, la pedagogía y la tecnología. Por su formación académica y su experiencia laboral en educación, el conocimiento de contenido (CK) y la pedagogía (PK) son los elementos que puede aportar el docente; y el conocimiento tecnológico (TK), tecnología de hardware, está a cargo del investigador, por su formación académica y práctica en el campo de la electrónica (Tourón, 2016).

Con esta visión y tras la necesidad de generar una propuesta de mediación tecnológica apoyada en la cultura Maker, en búsqueda de la mejora de la práctica docente de la enseñanza de las materias de ciencias y tecnología en secundaria, es que se implementa un diseño metodológico de investigación-acción. Se identifica en Katayama (2014), Hernández et al. (2010) y Martínez (2007) como el diseño adecuado para la investigación donde se busca una mejora de la realidad social, e indican que en este diseño el conocimiento se construye por la práctica e involucra la transformación y la mejora de una realidad educativa, donde se unen las capacidades, los conocimientos prácticos, las vivencias y las habilidades de los participantes e interactúan de manera constante y cíclica.

Específicamente se planteó una modalidad de investigación-acción de tipo práctico, al plantear el protagonismo en el docente, quien identifica su particular realidad social para desarrollar una estrategia propia de mejora de su práctica docente (Martínez-Salgado, 2012).

Además Martínez (2007) nos deja ver que, para un escenario educativo donde se cuenta con docentes participantes que toman el rol de investigadores de su realidad, puede existir un docente investigador externo quien introduce una propuesta de cambio para trabajar el proceso investigativo, el cual toma la postura de investigador cooperador, quien en este caso y como se menciona en Hernández et al. (2010), este investigador se une con sus capacidades, habilidades y experiencias con el resto de los sujetos que participan, para entrar en conjunto en una dinámica de acción y reflexión.

Esta investigación buscó proveer al docente de una experiencia de su acción, situación que se consideró importante y que orientó la recolección de los datos de esta investigación bajo un enfoque biográfico, con el cual se centró la atención en los relatos de la experiencia vivida por los docentes; al concebirlo como el actor y el productor de toda su experiencia (Cornejo, 2006).

Para contar con al menos un aspecto homogéneo de los docentes y una búsqueda relativamente rápida de éstos, se focalizó el esfuerzo y viabilidad para realizar la investigación en una muestra por criterio (Martínez-Salgado, 2012), con el fin de reducir la población a sólo los docentes interesados en integrarse en un proceso de mejora y reflexión de su práctica docente.

Respecto a la confiabilidad de la investigación, al ser de corte cualitativo se observó es dependiente del contexto. La validez de la investigación se consideró por elaboración o de constructo, por centrar la importancia en el diseño inicial y a partir de la reflexión de los hallazgos integrar o desechar elementos al diseño (Gil, 2011).

El esquema general, de este diseño de investigación-acción, consta de dos eventos, uno en cada ciclo escolar, con dos ciclos en cada uno, como se puede apreciar en la Figura 3, en la cual se describe el trabajo de campo de la investigación. 


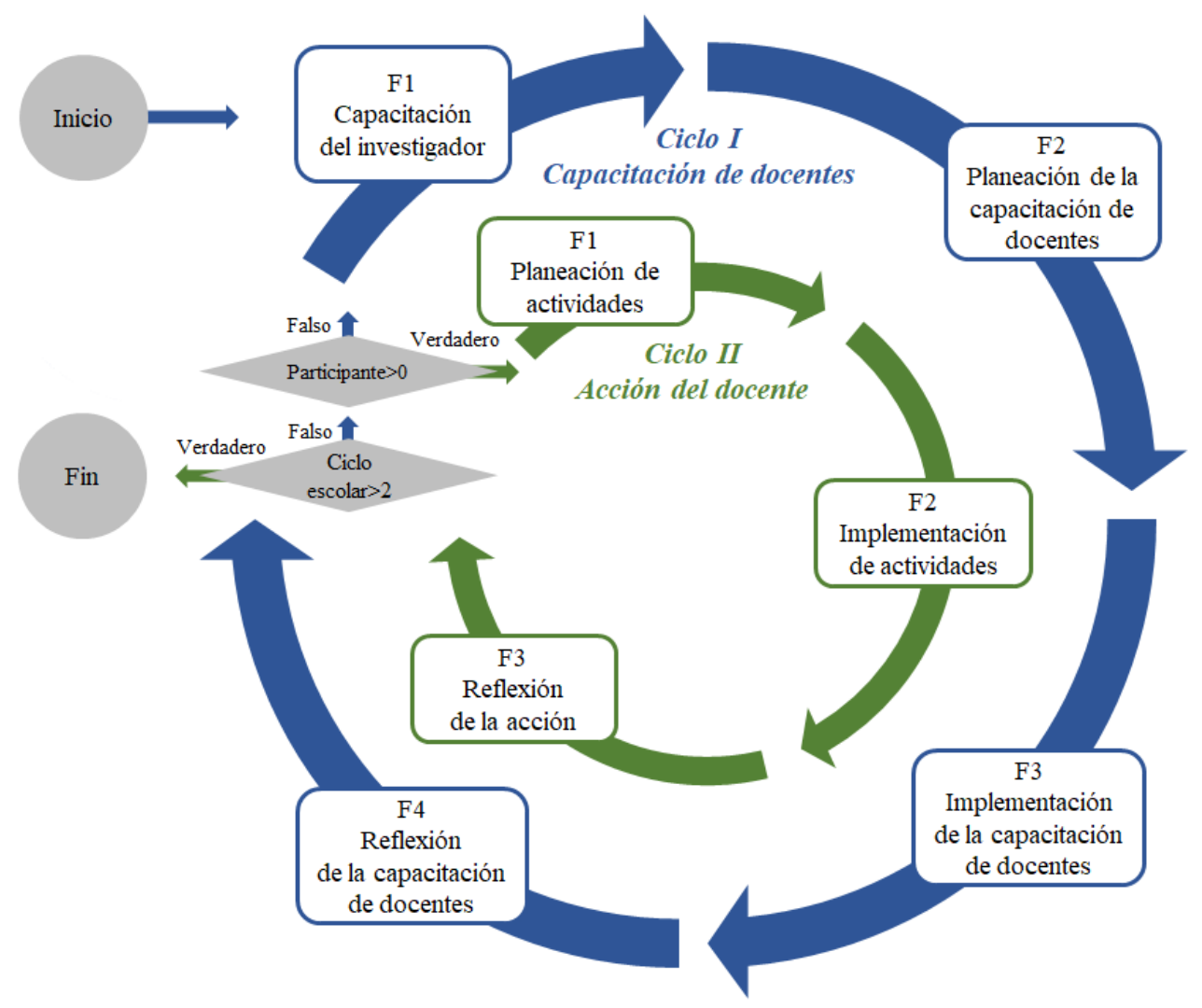

Figura 3. Esquema del trabajo de campo.

Se inicia con el Ciclo I, identificado como la capacitación de docentes, donde se plantea el proceso para buscar participantes al proyecto. Consta de cuatro fases, que empiezan con la capacitación del investigador, seguido de la planeación de la capacitación del docente, la implementación y al final la reflexión de la capacitación del docente. Una vez ejecutado este primer ciclo, si existen docentes interesados en continuar participando, se prosigue con el Ciclo II. De no existir docentes que deseen continuar participando, se repite el primer ciclo, dentro del primer evento hasta lograr al menos un participante para continuar al ciclo siguiente.

El segundo ciclo, Ciclo II, es identificado como la acción del docente, propiamente la intervención educativa por parte del docente, que consta de tres fases: planeación de la acción del docente, implementación de actividades en el aula y la reflexión de la acción. Una vez terminado el segundo ciclo se analizarán los datos obtenidos y se dará paso a un segundo evento en el siguiente ciclo escolar, donde se repite nuevamente todo el proceso como fue descrito, con otros profesores interesados. Al terminar el segundo evento se finaliza el trabajo de campo.

De acuerdo con Colmenares y Piñero (2008) el diseño de la investigación-acción se trata de desarrollar un proceso sistematizado que se basa en la recopilación y análisis de evidencias que resultan de la experiencia vivida por los participantes, enfatizando que es importante que las técnicas que se emplean respondan a un proceso planificado e intencionado de las acciones a seguir, desde el punto de vista investigativo y desde la perspectiva formativa de quienes participan. De esta manera toda la información que se recopile proveerá de elementos de reflexión sobre las acciones implementadas y los cambios alcanzados por los participantes les permitirán tomar decisiones respecto a los posibles ajustes o reconsideraciones. 
En las Tablas 1 y 2 se muestran los instrumentos que se emplearon en los ciclos I y II respectivamente, ahí se indica la técnica, el instrumento, el sujeto participante, el momento en que se aplica y los medios con los que se recolecta la información.

Tabla 1: Técnicas de recolección de datos aplicadas en el ciclo I.

\begin{tabular}{lllll}
\hline Técnica & Instrumento & Sujeto & Tiempo & Medio \\
\hline Observación participante & Bitácora & Investigador & Durante & $\begin{array}{l}\text { Notas de campo, grabación } \\
\text { de audio y fotos }\end{array}$ \\
Encuesta & Cuestionario & Docente & $\begin{array}{l}\text { Al inicio, durante } \\
\text { y al final } \\
\text { Formulario Google }\end{array}$ & Gl final \\
Entrevista en profundidad & $\begin{array}{l}\text { Entrevista } \\
\text { abierta }\end{array}$ & Docente & \begin{tabular}{l} 
Grabión de audio \\
\hline
\end{tabular}
\end{tabular}

Tabla 2: Técnicas de recolección de datos aplicadas en el ciclo II.

\begin{tabular}{|c|c|c|c|c|}
\hline Técnica & Instrumento & Sujeto & Tiempo & Medio \\
\hline Observación participante & Bitácora & Investigador & Durante & $\begin{array}{l}\text { Notas de campo, grabación } \\
\text { de audio y fotos }\end{array}$ \\
\hline Observación participante & Bitácora & Alumnos & Durante & Registro anecdótico \\
\hline Observación participante & Bitácora & Docente & Durante & Registro anecdótico \\
\hline Entrevista en profundidad & Entrevista abierta & Docente & Al final & Grabación de audio \\
\hline
\end{tabular}

Las encuestas del Ciclo I y los ítems de las entrevistas abiertas tanto del Ciclo I como del Ciclo II se presenta en el Apéndice A.

En la Figura 4, tenemos un esquema donde se muestra en secuencia vertical, de arriba hacia abajo, las técnicas empleadas en la investigación, se integraron las técnicas de recolección de datos antes mencionadas, las de codificación y las de análisis.

La recolección de los datos se realizó bajo enfoque biográfico, que de acuerdo con Cornejo (2006) plantea dicho enfoque porque se realiza una secuencia de actividades que permiten al investigador proveer de experimentación al docente, y así hacer surgir de la experiencia vivida elementos significativos para esta investigación. De esta manera se concibe al docente como el actor y el productor de toda su experiencia. También reconoce que el docente sabe más y mejor sobre su propia vida.

La acción de codificar la información consistió en establecer unidades de significado, de acuerdo con Massot et al. (2009). Estas unidades se identifican en la codificación como categorías. Por consiguiente, el proceso de categorización se realizó dando lectura a los documentos, uno a uno, con el fin de encontrar situaciones particulares en los relatos para generar dichas unidades de significado. 


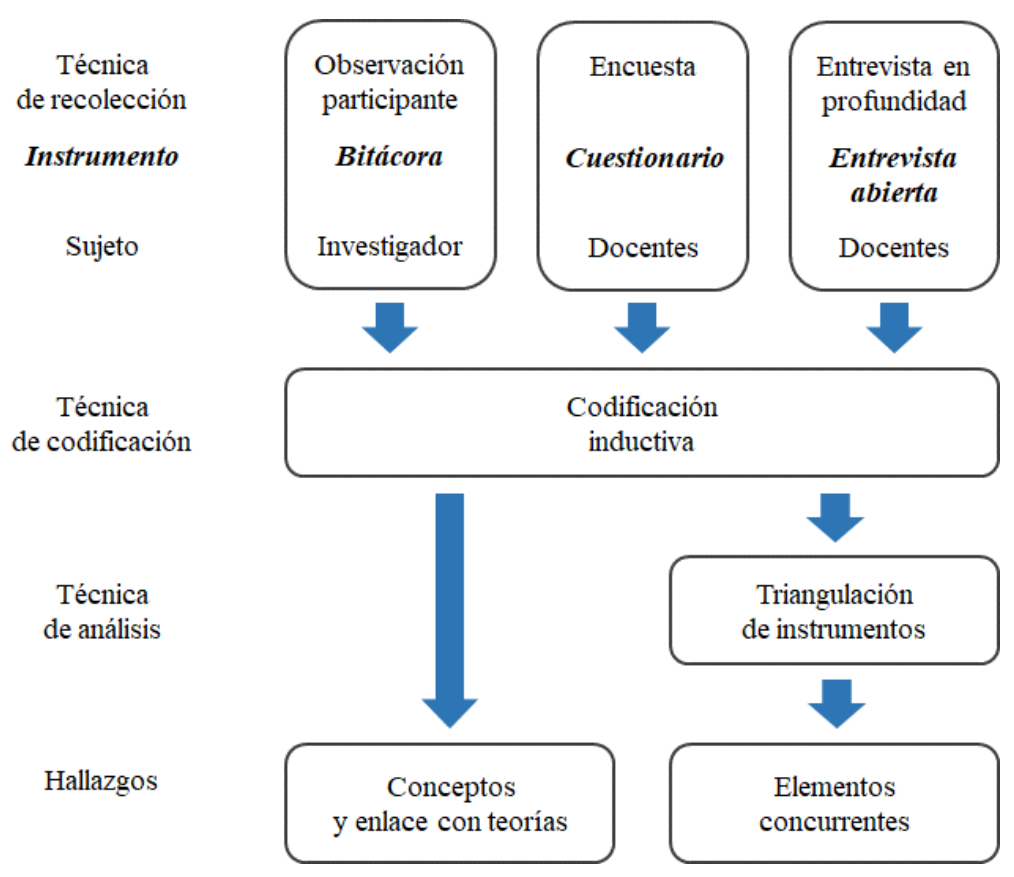

Figura 4. Técnicas empleadas en el ciclo I.

Con lo anterior y de acuerdo con Fernández (2006) y Massot et al. (2009), se apoya la idea de encontrar significados y se identifica la codificación de forma inductiva para esta investigación. No se contó con codificación previa a la recolección de los datos, sin embargo, de esos datos surge la información que dará pauta a la generación de los códigos que lo representen.

La codificación se realizó con la estructura empleada en el método de teoría fundamentada, método de investigación cualitativa considerado en Dorio et al. (2009) por orientarse a comprender el fenómeno; que de acuerdo con Strauss y Corbin (2002), esto sucede al establecer una estructura para la reducción de la información en función de tres niveles de codificación: abierta, axial y selectiva. Estos niveles se desarrollan desde lo sucedido en el campo hasta la descripción en términos de conceptos. Visto desde la perspectiva del investigador, servirá para identificar las teorías contempladas en esta investigación dentro de estos códigos.

Se realizo de la siguiente manera: las expresiones registradas se seleccionaron y se agruparon para crear conjuntos de expresiones, donde cada conjunto representó un argumento particular emergente, que dio lugar a la codificación selectiva. Después se analizaron estos argumentos con el fin de lograr sentido para después contextualizarlos en un concepto, se detectó así el concepto al que refiere dicho conjunto de expresiones, con lo que se formó la codificación abierta. Cada uno de los grupos de expresiones comúnmente solo trata alguna parte del concepto que se visualiza, por lo cual fue necesario ubicar cada uno de estos grupos en el caso adecuado o elemento particular argumentado en el concepto, identificando así la codificación axial.

Una vez desarrollado lo anterior se empleó la técnica de triangulación de instrumentos, con la que se lograron hallazgos en términos de los elementos concurrentes de esta parte del trabajo de campo.

De manera similar qué en la figura 4, se presentan en la figura 5 las técnicas empleadas ahora para el Ciclo II.

Respecto a la recolección de los datos se tuvo la observación participante y la entrevista en profundidad, con los instrumentos bitácora y entrevista abierta respectivamente, estos se aplicaron a los sujetos involucrados en la acción del docente; el investigador, el docente y sus estudiantes. Esto también bajo un enfoque biográfico. Una codificación inductiva, estructurada bajo el criterio 
de la teoría fundamentada, pero ahora una triangulación de sujetos, que dio lugar a la obtención de hallazgos en términos de conceptos, su enlace con las teorías y los elementos concurrentes que resultaron de este ciclo II.

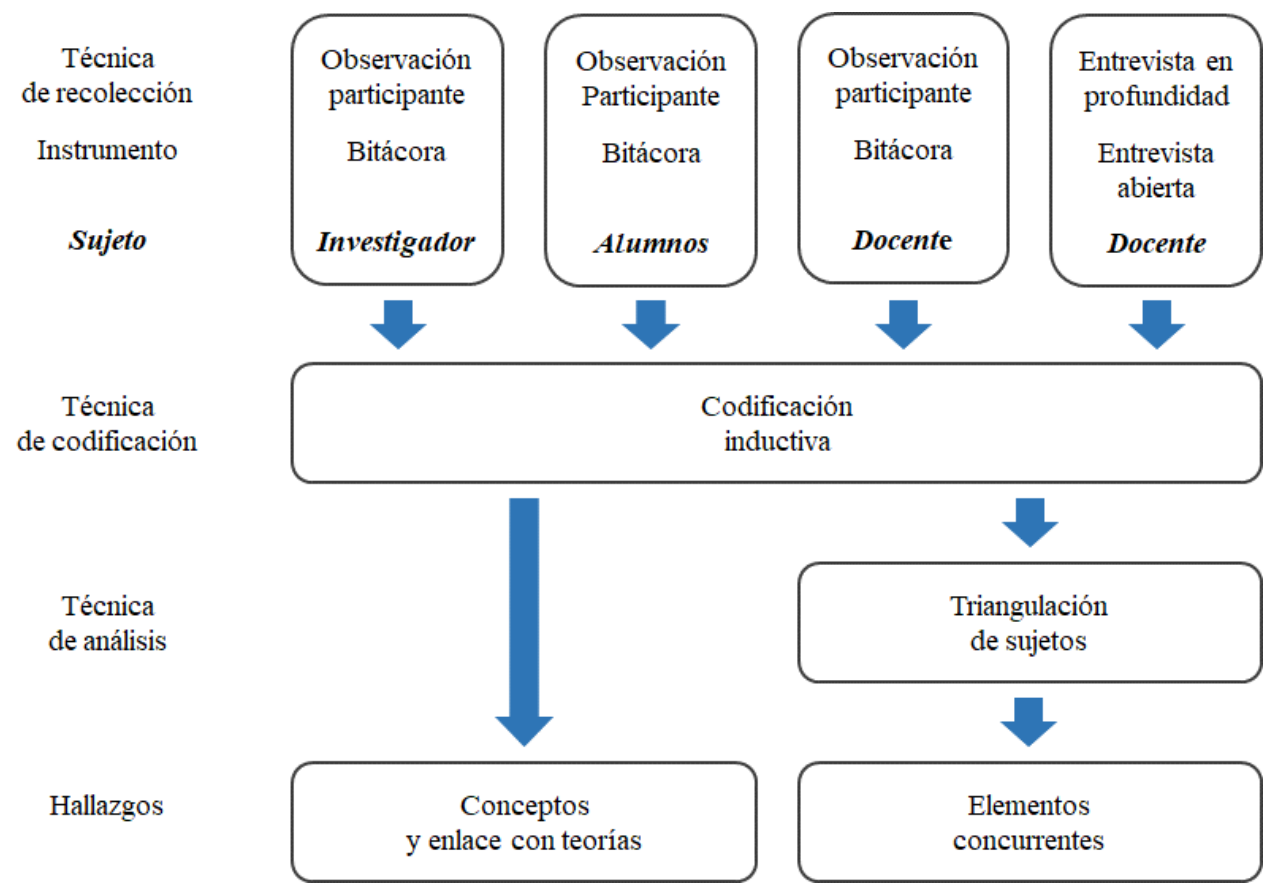

Figura 5. Técnicas empleadas en el ciclo II.

Según Hidalgo (2005) la triangulación está concebida como un modo de proteger las posibles tendencias del investigador, al confrontar los relatos de los diferentes informantes involucrados en la investigación. Al comparar las diversas apreciaciones se podrá observar dónde concuerdan, obteniéndose así una solidez en los hallazgos.

Massot et al., (2009) agrega se logra un significado mayor con la combinación de varias metodologías de triangulación en el estudio.

Retomando lo descrito en las figuras 4 y 5 respecto a la triangulación de instrumentos y de sujetos respectivamente, en Cohen y Manion (2002) éstas se identifican como triangulación metodológica entre los métodos. De tal manera que, en el Ciclo I se permitió validar en conjunto con el investigador el punto de vista del docente, sujeto de estudio, con diferentes instrumentos en diferentes momentos. Y en el Ciclo II se validó con el investigador, los puntos de vista de los diferentes informantes involucrados; con el docente y sus estudiantes.

Tomando en cuenta lo anterior, se tuvo una triangulación valida al tener dos o más argumentos de los diferentes instrumento o sujetos según el caso, que coincidieron en un código, reduciendo así la información y logrando mayor solidez en este código.

El haber diseñado un trabajo de campo donde se replicó el proceso con un segundo evento con diferentes participantes, se consideró la aplicación de la triangulación dentro de los métodos, acción que de acuerdo con Cohen y Manion (2002) consistió en identificar los elementos concurrentes de ambos eventos, para así obtener una mayor solidez en los códigos.

Se tuvo como resultado una combinación de metodologías de triangulación como se muestra en la Figura 6. 


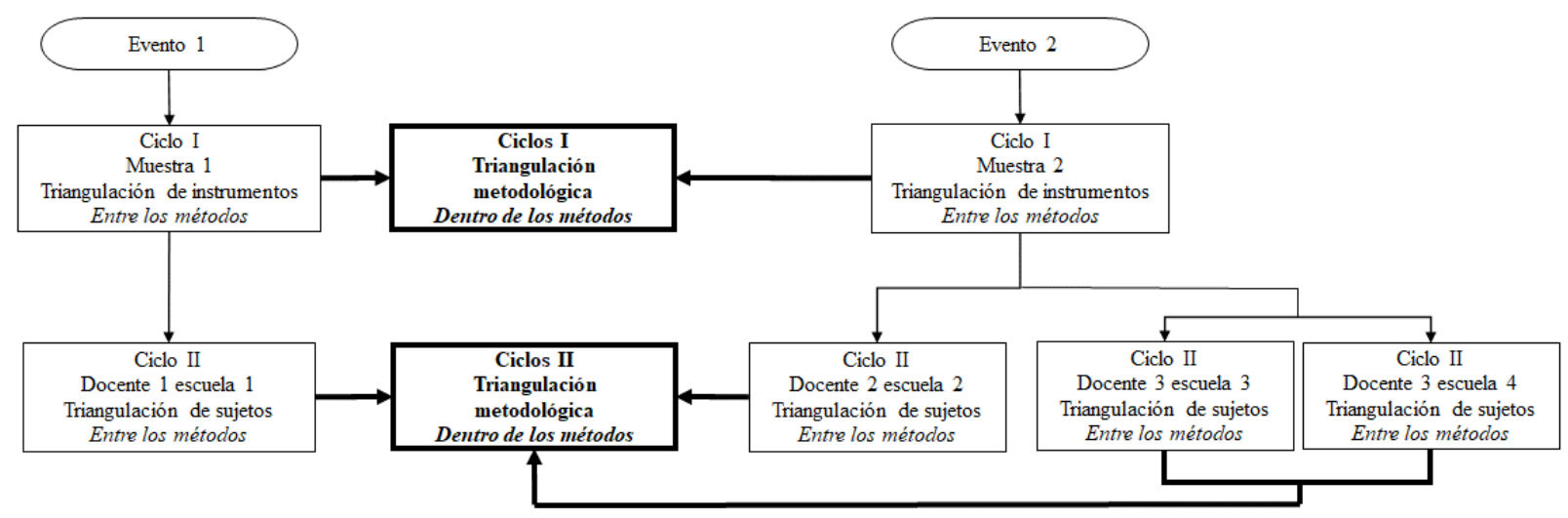

Figura 6. Combinación de metodologías de triangulación.

Para llevar a cabo todo el análisis, la información obtenida de los instrumentos aplicados en esta investigación, se transcribieron las grabaciones de audio y textos escritos en un procesador de texto, esto permitió trabajar la información de entrada en un sólo formato, en el software de análisis de datos cualitativos asistido por computadora MAXQDA (2018).

Los hallazgos que se obtuvieron están dentro de los siguientes aspectos: los conceptos derivados de los argumentos de los participantes, el enlace de la codificación con las teorías del Construccionismo y la Andragogía, y la concurrencia dentro de los métodos.

\section{Resultados}

\subsection{El trabajo de campo}

El primer evento del trabajo de campo se desarrolló en el periodo escolar 2017-2018 (Ver Tabla $3)$.

Tabla 3: Periodo del evento 1.

\begin{tabular}{ccll}
\hline Ciclo & Fase & \multicolumn{1}{c}{ Descripción } & \multicolumn{1}{c}{ Fechas } \\
\hline I & 1 & Capacitación del investigador & Noviembre - diciembre 2017 \\
& 2 & Planeación de la capacitación de los docentes & Enero 2018 \\
& 3 & Implementación de la capacitación de los docentes & Febrero 2018 \\
& 4 & Reflexión de la capacitación de los docentes & Marzo 2018 \\
\hline II & 1 & Planeación de la acción del docente & Marzo 2018 \\
& 2 & Implementación de la acción del docente & Abril - mayo 2018 \\
& 3 & Reflexión de la acción del docente & Mayo 2019 \\
\hline
\end{tabular}

Lo relativo al trabajo investigativo tanto del Ciclo I como del Ciclo II del primer evento fueron publicados en Domínguez-González et al. (2018) y Domínguez-González et al. (2019) respectivamente.

El segundo evento del trabajo de campo se desarrolló en el periodo escolar 2018-2019, en las fechas descritas en Tabla 4. 
Tabla 4: Periodo del evento 2.

\begin{tabular}{ccll}
\hline Ciclo & Fase & \multicolumn{1}{c}{ Descripción } & \multicolumn{1}{c}{ Fechas } \\
\hline I & 1 & Capacitación del investigador & Marzo 2018 \\
& 2 & Planeación de la capacitación de los docentes & Agosto 2018 \\
& 3 & Implementación de la capacitación de los docentes & Septiembre 2018 \\
& 4 & Reflexión de la capacitación de los docentes & Marzo 2020 \\
\hline II & 1 & Planeación de acción de los docentes & Octubre - noviembre 2018 \\
& 2 & Implementación de la acción de los docentes & Noviembre 2018 - abril 2019 \\
& 3 & Reflexión de la acción de los docentes & Mayo 2020 \\
\hline
\end{tabular}

El inicio de este segundo evento comenzó con el Ciclo I con la capacitación del investigador, en esta fase F1 se tomaron en cuenta las observaciones y reflexiones de la capacitación de los docentes del evento anterior (Domínguez-González et al., 2018). A partir de lo anterior se decidió modificar la fecha del taller de capacitación al inicio del periodo escolar 2018-2019 para así permitir a los participantes la alternativa de modificar su plan de trabajo anual e incluir su propuesta de cambio tras la capacitación tomada. Asimismo, reubicar las mesas de trabajo del taller en formato de islas y sustituir el tablero de circuitos electrónicos (breadboard) por uno más grande para lograr mejorar su desempeño. Además, invitar a la maestra que llevó a cabo el Ciclo II, acción del docente en el aula del evento anterior (docente 1 escuela 1), para compartir su experiencia a los demás profesores.

En la siguiente fase, planeación de la capacitación de los docentes, se tomó la misma planeación del primer evento con un taller con enfoque práctico promovido como Taller docente Maker, con la finalidad de entender la cultura Maker y exponer a los docentes a la experiencia de aprender nuevas cosas con tecnología (hardware).

Se llevó a cabo en una sala amplia, de cincuenta metros cuadrados aproximadamente, con proyector de video, pizarrón, conexión inalámbrica de internet, conexiones eléctricas, mesas y sillas que se podían mover en el momento que fuese necesario. Esto permitió en cada sesión modificar la ubicación de sillas y mesas para tener dos áreas integradas en la misma sala. En un área se montaba de forma tradicional para a la proyección de diapositivas y videos; y en la otra, una distribución de cuatro islas, donde se llevó a cabo todo el aspecto práctico del taller. Además, se tuvo una sala de receso con sillones y mesa de centro, que permitió realizar charlas amenas con los docentes. También se contó con herramientas como desarmadores, pinzas, cautín y taladro que se llevaron al área de trabajo.

Los participantes cubrieron el costo de recuperación para los materiales que emplearon y se les solicitó traer una computadora portátil. Se impartió el taller en cuatro sesiones en un total de veinte horas, cinco horas por cada sesión por la mañana, en sábados para no interferir en las actividades laborales de los maestros.

Todas estas consideraciones se tuvieron con la finalidad de obtener mayor poder de convocatoria, que se difundió a través de redes sociales, la radio y con visitas a algunas escuelas secundarias de la localidad.

El objetivo del taller de capacitación fue: los participantes conocerán la cultura Maker, así como algunas teorías y técnicas para aplicar en su actividad docente, desarrollando ejercicios prácticos usando componentes electrónicos y Arduino, concluyendo en la construcción de un robot con Arduino. Los temas fueron: Movimiento Maker, construccionismo, cultura Maker en educación, circuito electrónico, material y montaje electrónicos, soldadura, tendencias de la cultura Maker, Arduino, ejercicios y proyectos, el triángulo interactivo de enseñanza y aprendizaje, design thinking, modelo tecno pedagógico TPACK, aprendizaje cooperativo, intercambio de experiencias docentes y la participación de la maestra que realizó intervención educativa en el aula. 
La implementación de la capacitación de los docentes contó con profesores entre veintisiete y cincuenta años con nivel escolar en su mayoría de licenciatura que imparten clase mayormente en escuela pública entre otros datos. En la Tabla 5 se muestran los participantes que se tuvieron en el Ciclo I del evento 2.

Tabla 5: Muestra del segundo taller de capacitación.

\begin{tabular}{|c|c|c|c|c|c|c|}
\hline Participante & Género & Edad & Nivel de estudios & Escuela & $\begin{array}{l}\text { Materia que } \\
\text { Imparte }\end{array}$ & $\begin{array}{c}\text { Experiencia } \\
\text { en años }\end{array}$ \\
\hline 1 & Hombre & 46 & $\begin{array}{l}\text { Lic. Enseñanza en } \\
\text { ciencias naturales }\end{array}$ & Pública & Física & 20 \\
\hline 2 & Hombre & 50 & $\begin{array}{l}\text { Lic. Enseñanza en } \\
\text { ciencias naturales }\end{array}$ & Pública & Química & 21 \\
\hline 3 & Hombre & 28 & $\begin{array}{l}\text { Lic. Enseñanza en } \\
\text { física }\end{array}$ & Pública & Física, Matemáticas & 5 \\
\hline 4 & Mujer & 40 & $\begin{array}{l}\text { Lic. Enseñanza en } \\
\text { ciencias naturales }\end{array}$ & Pública & Ciencias & 13 \\
\hline 5 & Hombre & 27 & Lic. Mecatrónica & Pública & $\begin{array}{l}\text { Taller de } \\
\text { electricidad }\end{array}$ & 3 \\
\hline 6 & Hombre & 44 & Lic. Computación & Pública & Informática & 15 \\
\hline 7 & Mujer & 40 & $\begin{array}{l}\text { Maestría en educación } \\
\text { en ciencias }\end{array}$ & Privada & $\begin{array}{l}\text { La Naturaleza de la } \\
\text { ciencia, Estudio del } \\
\text { Mundo Natural. }\end{array}$ & 15 \\
\hline 8 & Mujer & 45 & $\begin{array}{l}\text { Lic. Enseñanza en } \\
\text { ciencias naturales }\end{array}$ & Pública & Ciencias I y II & 15 \\
\hline 9 & Mujer & 50 & Lic. Computación & Privada & $\begin{array}{l}\text { Computación y taller } \\
\text { de ciencias }\end{array}$ & $<1$ \\
\hline 10 & Mujer & 40 & Ingeniería industrial & Pública & Matemáticas & 10 \\
\hline
\end{tabular}

La reflexión de la capacitación del docente se obtuvo a partir de los argumentos otorgados por los propios maestros, a través del análisis de los grupos de los datos específicos de esta sección del proyecto, apoyado con MAXQDA (2018).

Al hacer el análisis de datos del Ciclo I (vía instrumentos aplicados) de este evento 2, se identificaron ciento veintiocho relatos que refirieron a un conjunto de trece conceptos: actitud, Andragogía, apropiación de la cultura Maker, computación, cualidades humanas, interacción social, motivación, práctica docente, recursos tecnológicos, sentimientos, serendipia, tutoría entre pares y visión.

Respecto a la triangulación de instrumentos se tuvo una identificación de ocho conceptos, de catorce categorías de noventa y dos expresiones. Esta sección del proceso investigativo nos llevó a encontrar argumentos de validez al ubicar coincidencias de los relatos en al menos dos de los tres instrumentos aplicados, que ofrecen hallazgos en relación con lo sucedido tras la experiencia ofrecida a los docentes. Aquí se identificó: una actitud colaboradora entre docentes; un interés del docente por aprender temas de aplicación inmediata para su práctica docente; preferencia por aprender a través de la práctica; interacción entre docentes al compartir su experiencia; entusiasmo al aprender nuevas cosas, lo que motivó al docente a descubrir la aplicabilidad de la cultura Maker en la educación; lo acontecido implicó la reflexión del docente sobre la cultura Maker en su práctica docente; se presentó el aprendizaje cooperativo con la tutoría entre pares, al apoyarse el docente con otros por su experiencia; las expectativas del docente por aprender cosas nuevas para su práctica docente; la utilidad de la cultura Maker para la solución de problemas; lograr un aprendizaje ligado a las emociones de sus alumnos; y finalmente, una expectativa de desanimo, al no contar con el apoyo de la dirección escolar por limitarlo a hacer cambios, bajo el argumento de la falta de recursos económicos. 
Para al Ciclo II se contó con dos participantes, éstos se muestran en la Tabla 5 en color gris como los participantes 2 y 5 . El primero se identificó como docente 2 en escuela 2, este maestro imparte clases en la sección de secundaria de un centro escolar en zona conurbada de Puebla, México. El segundo profesor se identificó como docente 3 en escuela 3 y 4, éste imparte clases en dos escuelas secundarias técnicas en los turnos matutino y vespertino respectivamente, en Puebla, México. Ambos docentes consideraron cambiar la dinámica que venían realizando en el aula: el primer docente planeo su intervención en el aula en el segundo trimestre del periodo escolar 2018-2019, en el curso extracurricular club de ciencias; el segundo profesor aplicó en el tercer trimestre del mismo periodo escolar, en el club de tecnología. Ambos lo llamaron actividad de la cultura Maker, que consistió en la construcción de un robot con Arduino. Esta actividad se deriva del mismo proyecto que desarrollaron en la etapa de su capacitación.

Con una propuesta para ser implementada en el aula, realizaron cada uno por su parte la gestión académica y de recursos en sus respectivos centros de trabajo. Se obtuvieron los permisos de sus autoridades y el conocimiento informado de los padres de familia. La propuesta consideró usar herramientas: cautín para soldar componentes electrónicos, desarmadores y pinzas para armar circuitos, y el taladro para realizar orificios que sirvieron para el montaje de componentes.

El primer docente planeó que las actividades de sus alumnos se realizaran de manera individual. El aula fue un laboratorio de ciencias que contaba con ocho mesas con bancos y estuvo integrada por alumnos de los tres niveles escolares de secundaria.

El segundo docente planeó actividades para desarrollarse en equipos de dos alumnos, en ambas escuelas. Implementó la actividad en talleres: en la escuela 3 fue el taller de electricidad y en la escuela 4 fue el taller de electrónica. En ambos casos se trató de un espacio con mesas de trabajo y bancos, con grupos de estudiantes de tercer grado de secundaria.

La triangulación de sujetos del docente 2 en la escuela 2, tuvo como resultado la identificación de trece conceptos derivados de ciento seis relatos. Que nos indican que existió: una actitud colaboradora entre los alumnos; los alumnos tuvieron gusto por ensamblar componentes electrónicos; a otros alumnos se les dificultó dicho ensamble de componentes; las estudiantes mujeres mostraron mejor destreza en el ensamble de componentes electrónicos; el control del aula tuvo dificultades por la gran cantidad de alumnos, lo que ocasionó un problema respecto a la atención de las instrucciones del maestro; los alumnos aprendieron a través de lo que hicieron; existió interés por otros maestros por lo que hizo el docente en el aula; hubo entusiasmo de parte de los alumnos al hacer actividades prácticas; el docente reflexionó por la experiencia que tuvo en el aula; algunos alumnos no llevaban el material que se le pedía; el docente se apoyó con el proyector de video para dar instrucciones al grupo; los alumnos mostraron satisfacción por la actividad práctica y por lo que hicieron para culminar la tarea; el docente observó cambios positivos en los alumnos, particularmente de algunos que no lo esperaba; los alumnos lograron terminar la actividad gracias a la tutoría entre pares; y el docente tuvo como expectativa lograr buenos resultados de su acción.

Del docente 3 en la escuela 3, la triangulación de sujetos tuvo como resultado la identificación de dos conceptos, relacionados con: la apropiación de la cultura Maker al sentirse capaz de desarrollar su plan de acción; y el relativo al material electrónico que solicitó a los alumnos, por el hecho de que en repetidas ocasiones los alumnos no llevaron el material solicitado para las actividades.

Y referente a los resultados de la triangulación de sujetos de la acción del docente, del grupo de la escuela 4, se identificaron tres conceptos: recursos tecnológicos, sentimientos y tutoría entre pares, que referían a las siguientes categorías: la situación de insuficiencia de computadoras para el grupo; el docente empleó el proyector de video como recurso tecnológico; los alumnos mostraron satisfacción al realizar actividades prácticas; algunos alumnos mostraron apatía al ser 
indiferentes a la actividad y finalmente los alumnos lograron terminar las actividades gracias a la tutoría entre pares alumnos; sucedida tras la petición del docente que solicito a los alumnos sobresalientes apoyar a los demás compañeros.

\subsection{Los documentos}

Como resultado del trabajo de campo establecido en la sección 2 (ver Figura 3), se obtuvo una serie de documentos derivados de la recolección de los datos; dichos documentos y su origen, se presentan en la Tabla 6.

Tabla 6: Procedencia de los documentos.

\begin{tabular}{|c|c|c|c|c|c|}
\hline Evento & Ciclo & Instrumento & Sujeto & $\begin{array}{c}\text { Escuela donde } \\
\text { ocurre }\end{array}$ & $\begin{array}{l}\text { Número de } \\
\text { documentos }\end{array}$ \\
\hline \multirow[t]{7}{*}{1} & I & Bitácora & Investigador & Universidad & 1 \\
\hline & & Cuestionario & Docentes & Universidad & 6 \\
\hline & & Entrevista abierta & Docentes & Universidad & 5 \\
\hline & II & Bitácora & Investigador & 1 & 1 \\
\hline & & Bitácora & Alumnos & 1 & 1 \\
\hline & & Bitácora & Docente 1 & 1 & 1 \\
\hline & & Entrevista abierta & Docente 1 & 1 & 1 \\
\hline \multirow[t]{15}{*}{2} & I & Bitácora & Investigador & Universidad & 1 \\
\hline & & Cuestionario & Docentes & Universidad & 6 \\
\hline & & Entrevista abierta & Docentes & Universidad & 5 \\
\hline & II & Bitácora & Investigador & 2 & 1 \\
\hline & & Bitácora & Alumnos & 2 & 1 \\
\hline & & Bitácora & Docente 2 & 2 & 1 \\
\hline & & Entrevista abierta & Docente 2 & 2 & 1 \\
\hline & & Bitácora & Investigador & 3 & 1 \\
\hline & & Bitácora & Alumnos & 3 & 1 \\
\hline & & Bitácora & Docente 3 & 3 & 1 \\
\hline & & Entrevista abierta & Docente 3 & 3 & 1 \\
\hline & & Bitácora & Investigador & 4 & 1 \\
\hline & & Bitácora & Alumnos & 4 & 1 \\
\hline & & Bitácora & Docente 3 & 4 & 1 \\
\hline & & Entrevista abierta & Docente 3 & 4 & 1 \\
\hline Suma & & & & & 40 \\
\hline
\end{tabular}

\subsection{Los conceptos}

Los documentos se importaron a MAXQDA (2018) y se codificaron a partir de la creación de categorías, bajo los criterios de la teoría fundamentada propuesta en Strauss y Corbin (2002). En esta etapa se llevó a cabo la codificación abierta, axial y selectiva, descritas en la sección 2 . El resultado fue un total de ciento cincuenta y nueve categorías.

Siguiendo el proceso de análisis de datos, presentado en la sección 2, se identificaron diecisiete conceptos en el nivel de codificación abierta; lo anterior, a partir de mil siete expresiones, mismas que dieron lugar a la identificación de los siguientes conceptos: actitud, Andragogía, apropiación de la Cultura Maker, clima organizacional, computación, control de grupo, cualidades humanas, emociones, interacción social, motivación, práctica docente, recursos tecnológicos, sentimientos, serendipia, sinergia multidisciplinaria, tutoría entre pares y visión.

En la figura 7 se presenta la captura de pantalla de la matriz de códigos realizado en MAXQDA (2018), y se visualiza la cantidad de expresiones totales, así como por cada concepto. Resalta que el mayor número de expresiones se define por el concepto Cualidades humanas, formado con doscientos veintinueve expresiones. 


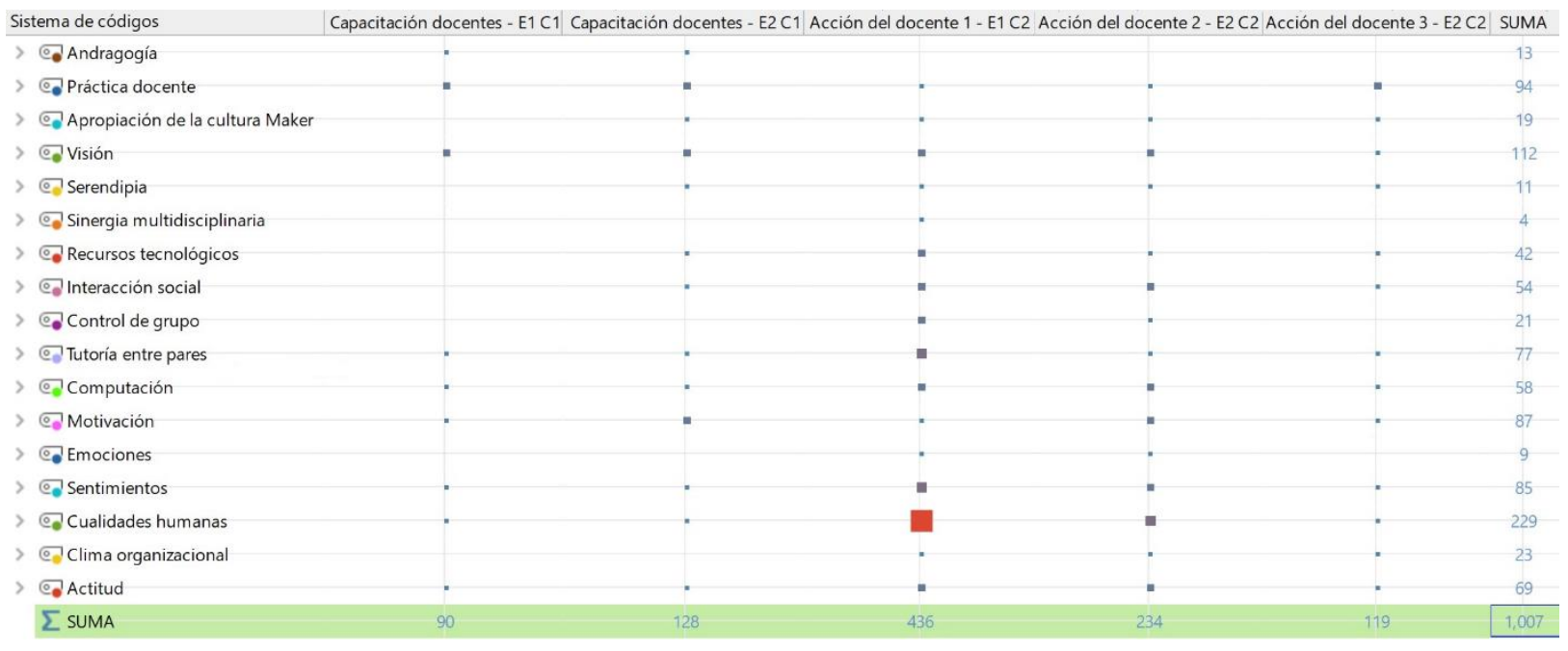

Figura 7. Matriz de códigos MAXQDA (2018).

\subsection{Concurrencias dentro de los ciclos}

Se describen las coincidencias del Ciclo I de ambos eventos, así como las coincidencias de Ciclo II también de ambos eventos. En la primera sección de nuestro proceso investigativo, respecto a la capacitación de los docentes, se tuvo una concurrencia en cinco conceptos que refieren a que: los docentes buscan aprender sobre la cultura Maker porque consideran que lo aplicaran en el corto plazo en la escuela; se logra de los maestros participantes una actitud colaboradora entre ellos; aparece de manera espontánea el aprendizaje cooperativo con la tutoría entre pares, al apoyarse uno con otros por su experiencia; los maestros logran entrar en un proceso reflexivo sobre la aplicabilidad de la cultura Maker en la educación, particularmente lo que implica para trabajar en un cambio para su práctica docente; se generó en los docentes la visión con la expectativa de apoyarse en dicha cultura para la solución de problemas, como estrategia didáctica para trabajar con sus alumnos en el aula.

En la segunda sección de nuestro proceso investigativo, respecto al acompañamiento de la acción del docente, se tuvo una concurrencia en seis conceptos que refieren a resultados respecto a los dos actores, el docente y los alumnos: el docente se apoyó con el proyector de video para lograr la comunicación de la instrucción a sus alumnos; se logró tener una actitud colaboradora entre los alumnos; para algunos estudiantes fue difícil el ensamble de componentes electrónicos; otros pocos alumnos no cumplían con el material solicitado, situación que causó retrasos en el desarrollo de las actividades planeadas por el maestro; algunos alumnos mostraron apatía al mostrar indiferencia por la actividad; la mayoría de los estudiantes expresaron satisfacción por hacer actividades prácticas y por lo que esto implicaba para culminar la tarea; se desarrolló el aprendizaje cooperativo con la tutoría entre pares alumnos, solicitado por el docente para terminar las actividades; el docente observó cambios positivos en los alumnos, además de otros cambios de alumnos que no se esperaba, específicamente por su interés, empeño y dedicación que no mostraban anteriormente.

\subsection{Enlace con las teorías}

Los aspectos de enlace de la codificación obtenida con la teoría del construccionismo fueron el aprendizaje y la socialización, dado que al ofrecer al alumno de una experiencia significativa para el aprendizaje a través de compartir conocimiento y desarrollarse en un contexto social (Tesconi, 2015).

Los comportamientos mostrados por los docentes en el proceso de apropiación de la cultura Maker, seguida de la toma de decisión para modificar su práctica docente, los vemos explicados 
en la Andragogía, disciplina que ayudó a entender el aprendizaje del adulto, en este caso el docente (Walker y Montero, 2004).

Considerados como aspectos de enlace con la andragogía al aprendizaje de temas relevantes, la aplicación útil e inmediata, la experiencia del docente y la retención diferente. La pertinencia de las teorías antes mencionadas se fue comprobando conforme se desarrolló el proceso de investigación, propiamente en el análisis de los datos, con la identificación de las categorías que hacen referencia a algún aspecto de la teoría, creando así un enlace de las teorías descritas con la codificación relacionado con el trabajo de campo y que concurre con la triangulación dentro de los métodos, que se muestra en la Tabla 7.

Tabla 7: Aspectos de enlace con las teorías.

\begin{tabular}{|c|c|c|}
\hline Teoría & Aspecto de enlace & Codificación: abierta/ axial / selectiva \\
\hline \multirow[t]{2}{*}{ Construccionismo } & Aprendizaje & $\begin{array}{l}\text { Visión / Expectativas del docente / La cultura Maker para la solución } \\
\text { de problemas }\end{array}$ \\
\hline & Socialización & $\begin{array}{l}\text { Actitud / Colaboradora / Existe colaboración entre docentes } \\
\text { Tutoría entre pares / Alumnos / El docente pide a los alumnos a } \\
\text { apoyar a otros } \\
\text { Tutoría entre pares / Docentes / El docente se apoya con otro por su } \\
\text { experiencia }\end{array}$ \\
\hline \multirow[t]{2}{*}{ Andragogía } & $\begin{array}{l}\text { Aplicación útil e } \\
\text { inmediata }\end{array}$ & $\begin{array}{l}\text { Actitud / Colaboradora / Existe colaboración entre alumnos } \\
\text { Andragogía / Aprendizaje / Aplicación inmediata } \\
\text { Tutoría entre pares / Alumnos / Los alumnos lograron terminar la } \\
\text { actividad } \\
\text { Visión / Expectativas del docente / La cultura Maker para la solución } \\
\text { de problemas }\end{array}$ \\
\hline & $\begin{array}{l}\text { Experiencia del } \\
\text { docente }\end{array}$ & $\begin{array}{l}\text { Práctica docente / Reflexivo / Del docente para su práctica docente } \\
\text { Práctica docente / Reflexivo / Del docente sobre la cultura Maker en } \\
\text { la educación }\end{array}$ \\
\hline
\end{tabular}

\subsection{Docentes post trabajo de campo}

Con el fin de conocer las acciones tomadas por los docentes participantes después de su colaboración en el proyecto de investigación, en virtud de sus acciones inmediatas para el siguiente periodo escolar, se les pidió comentar qué planes tenían sobre su práctica docente. El docente 1 implementará el análisis de objetos técnicos con sus alumnos del próximo curso. La profesora acordó la metodología con la dirección de la escuela y los padres de familia, de quienes ya contaba con el apoyo para permitir a sus hijos llevar aparatos descompuestos al taller; con la ayuda del conocimiento compartido encontrado en internet se identificarían sus partes y se conocería su funcionamiento. Lo anterior, con la idea principal de conocer los diversos aparatos de uso cotidiano y que, en el mejor de los casos, los mismos alumnos los repararan.

El entusiasmo e identificación con la Cultura Maker del docente 2, como él lo expresa, lo llevó a realizar una dinámica de emprendimiento en el segundo trimestre del ciclo escolar 20192020. En ella, los estudiantes crearon una microempresa y lograron fabricar productos como jabón, aromatizante, gel para cabello, gel para manos, llaveros con estambre y otros con glicerina, fertilizantes orgánicos y químicos, etc.

En el caso del docente 3, nos indica que en la escuela 3, la nueva dirección no aprobó la continuidad de las actividades propuestas. Sin embargo, expresó que, ante dicha situación, encontrará otras formas de abordar los temas del taller con la ayuda de la Cultura Maker. En la escuela 4 sí contó con el apoyo de la dirección de la escuela y padres de familia para continuar con actividades similares. Hace énfasis en el gran potencial de la Cultura Maker respecto a que, con un simple ejercicio con luces, permite al alumno relacionarlo con elementos de su cotidianidad, como el semáforo. La escuela se encuentra en un crucero complejo y el docente 
observó a algunos alumnos detenerse a analizar el comportamiento y sincronía de las luces, para replicarlo en una maqueta como resultado de un ejercicio que llevó al alumno a desarrollar algo tangible y útil.

Estos resultados orientan a incluir un nuevo componente al esquema planteado como trabajo de campo, donde se tiene un nuevo ciclo desarrollado por el docente, ahora sin acompañamiento del investigador. El profesor planea su acción apoyada en la Cultura Maker y plantea así una forma diferente de enseñar; dicha planeación la lleva a cabo y reflexiona sobre su acción (ver Figura 8), a este ciclo autónomo, que esperamos no tenga fin, lo denominamos Ciclo III Práctica del docente Maker. Este ciclo es a lo que aspirábamos con esta investigación.

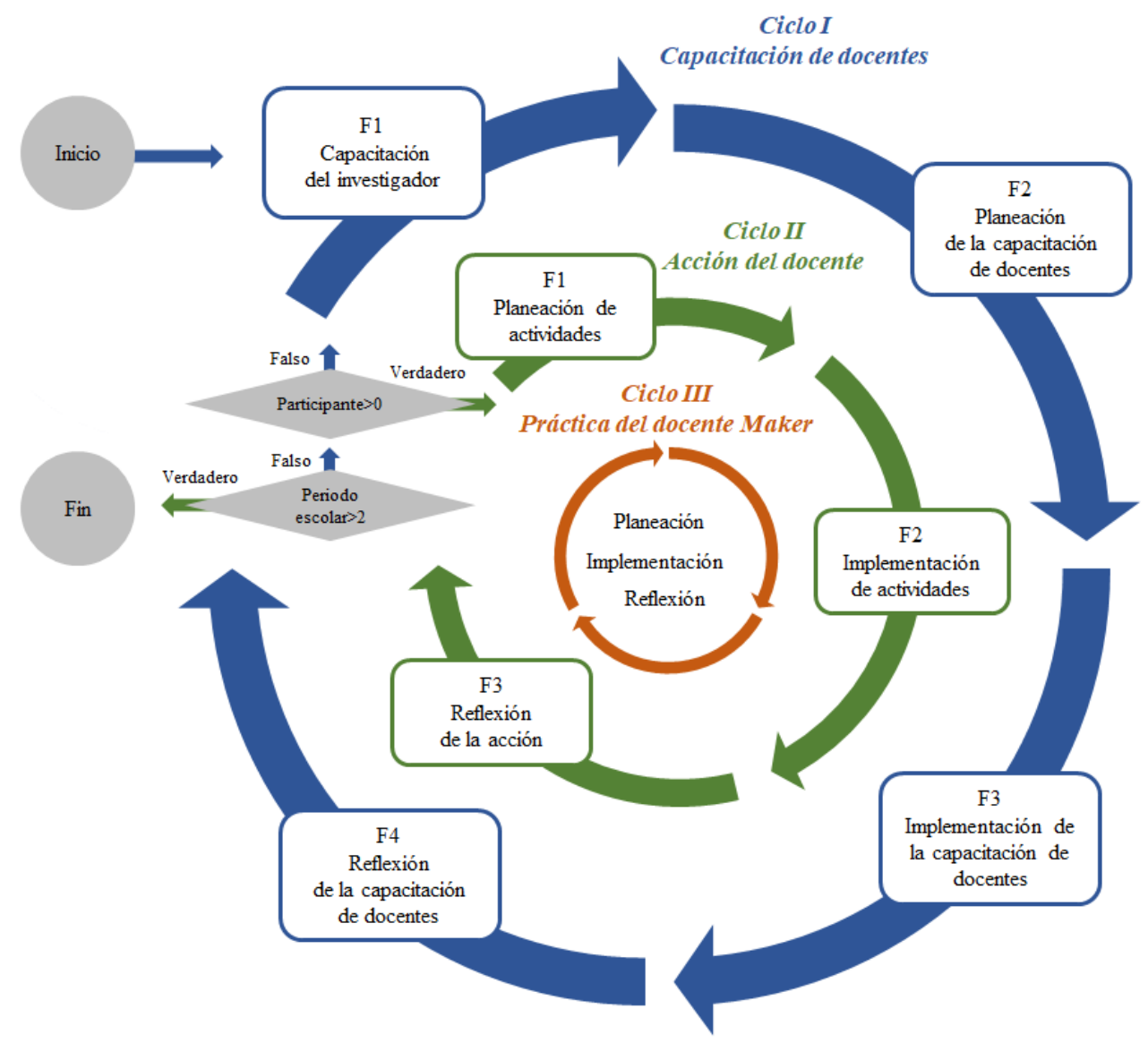

Figura 8. Nuevo esquema del trabajo de campo.

\subsection{Docentes sin acompañamiento}

Concluidos los Ciclos I, se contó con la participación de una profesora en el primer evento y dos profesores en el segundo evento, quienes decidieron participar llevando su acción al aula, permitiendo acompañarlos en dicha decisión. Sin embargo, en las entrevistas a profundidad aplicadas, se logró conocer el testimonio de otros profesores quienes, si bien no tuvieron el seguimiento posterior planteado en el proyecto de investigación, sí desarrollaron de manera independiente actividades en el aula. Estas actividades siguieron el esquema de planeación, implementación y reflexión de su acción en el aula, apoyada por la Cultura Maker. Es decir, llegaron al Ciclo III Práctica docente Maker (ver Figura 9). A continuación, describimos las experiencias que compartieron en la entrevista en profundidad del Ciclo I.

Una maestra del primer evento compartió su experiencia en la enseñanza de Excel. Para lograr el interés y aprendizaje del tema de tablas en Excel, consideró llevar a los alumnos diferentes piezas tipo LEGO y ligas de diferentes colores. Planteó una estrategia para hacer tablas en Excel 
mediante la clasificación de las piezas por colores y formas. Esto permitió a los estudiantes entender el proceso de contar y el uso del programa, por medio del uso de elementos tangibles y con significado para ellos. Incluso jugaron con las piezas después de terminar la actividad, como planteaba Papert en el Construccionismo, un ambiente lúdico. La docente comentó no haber abordado el tema de dicha manera anteriormente y señaló un cambio en su planteamiento y visión sobre la manera de enseñar.

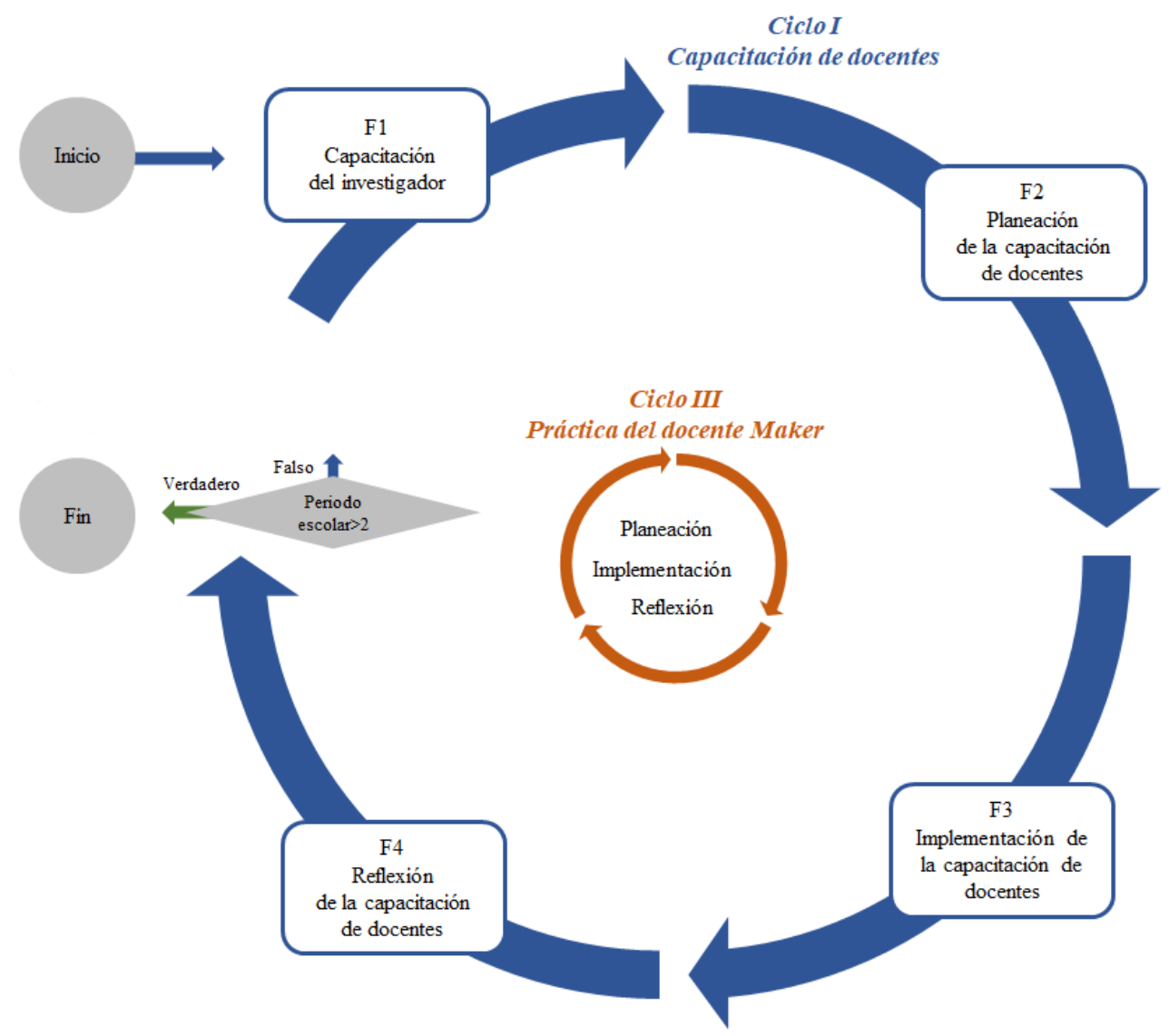

Figura 9. Acceso al Ciclo III de docentes sin acompañamiento.

Del segundo evento, una profesora y un profesor compartieron las actividades implementadas en sus escuelas después de participar en el taller de capacitación.

Ella no contaba con el apoyo, por parte de la dirección de la escuela, para realizar actividades que implicaran el uso de componentes electrónicos ni herramientas, esto debido a la falta de recursos económicos para la adquisición de los materiales. Sin embargo, construyó una pizarra didáctica con una tabla y pintura de pizarrón, junto a sus alumnos en clase de Matemáticas; la desarrolló pensando en la inclusión a la clase de una alumna que no puede oír ni hablar. Además de lograr dicha inclusión, obtuvo una participación mayor por parte del resto de los estudiantes en las clases. Con el artefacto creado, ayudó a sus alumnos a comprender el tema de fracciones. También comentó que otros profesores le pidieron compartir detalles de su acción.

Cabe resaltar la apropiación de la Cultura Maker por parte de la profesora, al hacer tecnología con sí misma, hacer con los demás y compartir sus hallazgos.

El otro profesor pidió a sus alumnos realizar un papalote en su clase de Física. La actividad implicaba investigar cómo se hacía aprovechando el conocimiento que se encuentra en internet, o con instrucciones dadas un familiar o amigos. El objetivo era hacerlo y volarlo en el patio de la 
escuela. El profesor informa no haber hecho este tipo de actividades con anterioridad. Considera que de este modo el alumno se divirtió y comprendió mejor el tema.

Otro artefacto elaborado fue un paracaídas con un muñeco. Nuevamente fue una actividad con la cual se divirtieron y aprendieron temas relativos a la materia de Física de secundaria. De este modo como lo menciona Badilla y Chacón (2004), se observan elementos del Construccionismo: objetos para pensar y entidades públicas.

Las acciones realizadas de manera autónoma por partes de los docentes permiten plantear la alternativa del desarrollo de la acción del docente sobre nuestro trabajo de campo y sin el acompañamiento del investigador. Se entiende que, de manera similar a la mencionada a la sección anterior, el docente realizó las etapas de planeación, implementación y reflexión de la acción del docente, pasando directamente al Ciclo III Práctica docente Maker (Figura 9). Evidenciando la apropiación de la Cultura Maker en su práctica docente, un hallazgo no esperado por los investigadores.

\section{Discusión}

Con esta investigación se explora una propuesta específica de mediación tecnológica apoyada en la cultura Maker, en la práctica educativa en secundaria, que tuvo como fin fomentar el cambio en la práctica docente del maestro de ciencias y tecnología. Los resultados dejan ver que la propuesta resultó ser factible, al lograr captar la participación de docentes, desarrollar el proyecto y la aplicación de los instrumentos planteados en el estudio.

Trabajar la recolección y el análisis de los datos bajo un enfoque biográfico y el desarrollo de una codificación inductiva, permitió estructurar la experiencia vivida por los actores en conceptos o temas que describen en un nivel abstracto el fenómeno social ocurrido. Y con la triangulación metodológica, se encuentra la fiabilidad al mostrar la congruencia entre los actores.

La participación por parte del investigador aportó el acercamiento de tecnología de hardware hacia el docente de secundaria. El trabajo cooperativo que realizó, dentro de la investigaciónacción, proporcionó un proceder más dinámico en la toma de decisiones para la acción y valorar los cambios realizados para la mejora de la práctica docente. Esta experiencia del investigador podría dar aportaciones valiosas a otros investigadores de otras disciplinas para incidir con nuevas recomendaciones tecnológicas, pedagógicas o del contenido para la formación y procesos de cambio del profesorado de secundaria.

Características como la edad, género y escolaridad no son factores que indiquen que un maestro se incline por el cambio de su práctica docente. Los maestros participantes no cuentan con formación normalista, son docente comprometidos con su profesión, sensibles a las necesidades e interés de sus alumnos, tienen disposición, son autoexigentes, son empáticos y sobresalientes en sus áreas de trabajo.

Con el desarrollo del trabajo de campo se infiere que al replicarlo en un contexto similar llevará a resultados semejantes.

Derivado del trabajo de campo tenemos una propuesta de mediación tecnológica para promover la apropiación de la Cultura Maker por parte del docente de Ciencia y Tecnología en Educación Secundaria, que se muestra en la Figura 10, donde se plantean los pasos que debe seguir un docente para lograr una acción en su aula. A diferencia de la Figura 3, aquí no se plantea la presencia del investigador.

Da inicio con la reflexión e identificación de las necesidades para la enseñanza, por parte del docente o el conjunto de profesores que coincidan en un interés común. El primer bucle consiste en la capacitación del docente. El segundo bucle está determinado por la acción del docente. 
Ambos se desarrollan en las fases de planeación, implementación y reflexión del docente o docentes, según sea el caso.

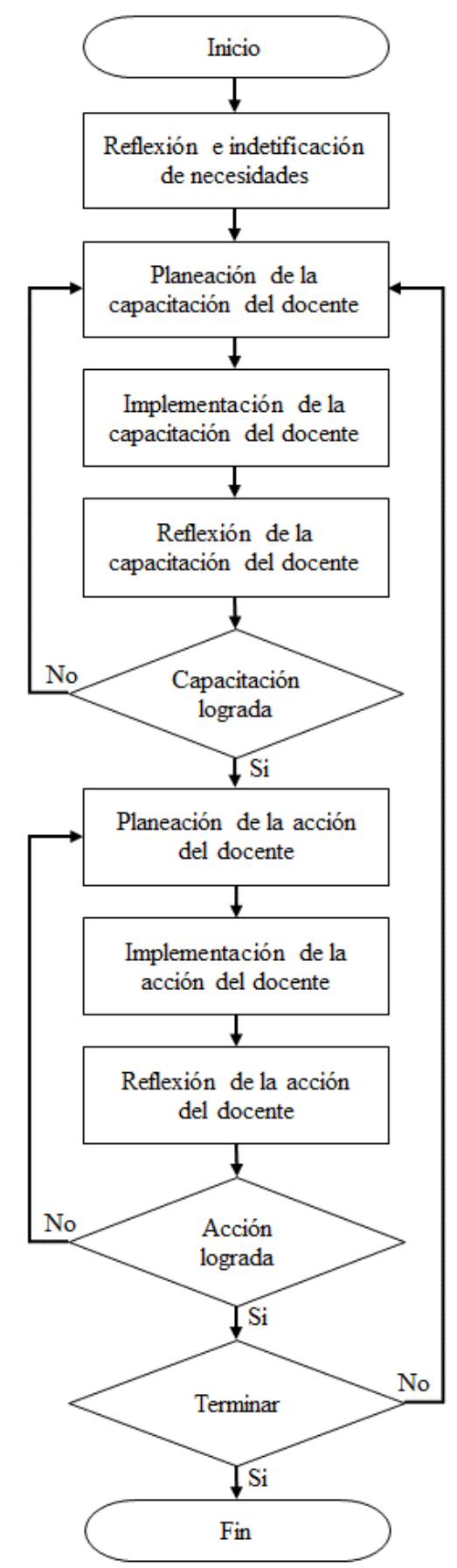

Figura 10. Acceso al Ciclo III de docentes sin acompañamiento.

El primer bucle inicia con la fase de planeación de la capacitación del docente, proceso donde gestiona forma, tiempo y espacio donde esta se llevará a cabo. Así, un primer momento está determinado por la búsqueda de otro u otros profesionistas en los que pueda apoyarse para acceder a los conocimientos tecnológicos que complementaran a los suyos. En un segundo momento planeará cuándo y dónde se realizará la capacitación del docente. 
La segunda fase es la implementación de la capacitación del docente. La tercera es la reflexión de su capacitación. Al finalizar esta fase, se determinará si se logró el objetivo o requiere replantear su capacitación.

Al terminar la capacitación, el proceso continúa con la acción del docente. Inicia con la planeación de su acción en el aula. En esta fase se desarrolla la gestión de tiempos, espacios y autorización de la dirección y padres de familia, según sea el caso. La siguiente fase de implementación de la acción del docente se desarrolla en el aula, donde se ejecuta lo planeado. Finaliza en una tercera fase de reflexión de la acción del docente. Esta fase sirve para determinan los aspectos de mejora para desarrollar una nueva acción en el aula o dirigir esfuerzos en replantear aspectos de su capacitación, si lo considera necesario. De esta manera, se visualiza la búsqueda de la mejora en cada uno de los bucles, tanto dentro de ellos como en todo el proceso de mediación tecnológica propuesto.

\section{Conclusiones}

La propuesta estimuló un cambio en la práctica docente del maestro, con la apropiación de las ideas y conocimientos de la cultura Maker: lo llevó a modificar la planeación de la enseñanza de su materia, con su propia estrategia de acción en el aula. Al acompañarlo en la implementación de dicha acción, se hacen evidentes los elementos de juicio de acción del docente, en función de sus habilidades, su experiencia en el aula, el protagonismo de su propia realidad, y porque que obedece a un problema que emana de su propio actuar. Las acciones del investigador, de estimular y acompañar, son situaciones de estudio para encontrar, bajo un enfoque inductivo de aplicación de instrumentos y análisis de los datos de la investigación cualitativa, las teorías que dan explicación a los hallazgos y la factibilidad de la propuesta. La cual resultó positiva en los dos trabajos de campo desarrollados, y al estar estructurado en un diseño de investigación-acción, las futuras aplicaciones pueden mejorarse, adaptándose a los contextos en que se desarrollen.

Se identifica que el docente adopta acciones de otros docentes si el proceso es claro, útil, lo motiva y es de interés para sus alumnos. También se observó que la capacitación del docente podría ser suficiente para algunos profesores, pero para otros no tanto.

\section{Agradecimientos}

Agradecemos el apoyo que nos brindó el CONACYT en el Doctorado en Sistemas y Ambientes Educativos de la BUAP. Así mismo, a todos los docentes que han participado de manera voluntaria en nuestro proyecto de investigación.

\section{Referencias}

Badilla, E. y Chacón, A. (2004). Construccionismo: objetos para pensar, entidades públicas y micromundos. Actualidades investigativas en educación, (1). https://www.redalyc.org/pdf/447/44740104.pdf [GS Search]

Cabero, J., Roig-Vila, R., y Mengual-Andrés, S. (2017). Conocimientos tecnológicos, pedagógicos y disciplinares de los futuros docentes según el modelo TPACK. Digital Education Review, 32(1). Recuperado de http://hdl.handle.net/11441/69058 [GS Search]

Cohen, L. y Manion, L. (2002). Métodos de investigación educativa. Editorial La Muralla. Madrid. ISBN 84-7133-565-4. 
Colmenares, A., y Piñero, M. (2008). La investigación acción una herramienta metodológica heurística para la comprensión y transformación de realidades y prácticas socioeducativas, Laurus, $\quad 14(27), \quad 96 \quad$ - $\quad 114 . \quad$ Recuperado de: http://www.digital.ciecas.ipn.mx/docs innova/pdfs/u4_2 investigacion-accion_educa.pdf. [GS Search]

Cornejo, M. (2006). El enfoque biográfico: trayectorias, desarrollos teóricos y perspectivas. $\begin{array}{llll}P S Y K H E, & \text { 15(1). } & \text { 95-106. } & \text { Recuperado }\end{array}$ https://scielo.conicyt.cl/scielo.php?script $=$ sci arttext\&pid $=$ S071822282006000100008\&lng=en\&nrm=iso\&tlng=en [GS Search]

Domínguez-González, M., Mocencahua-Mora, D., y Cuevas-Salazar, O. (2018). Taller Docente Maker para la enseñanza de ciencia y tecnología en la educación secundaria. Innovación, Tecnología y Liderazgo en los entornos educativos, pp. 169-179. Humboldt International University, EU. ISBN 978-0-9915776-7-5. [GS Search]

Domínguez-González, M., Mocencahua-Mora, D., y González-Calleros, J. (2019). Práctica docente apoyada en la cultura Maker para educación secundaria. Campus Virtuales, 8(2), 3546. [GS Search]

Dorio, I., Sabariego, M. y Massot, I. (2009). Características generales de la metodología cualitativa. En R. Bisquerra (ed.), Metodología de la investigación educativa, (pp. 275-292). Universidad de Barcelona. ISBN 978-84-7133-748-1.

Fernández, L. (2006). Cómo analizar datos cualitativos. Recerca. Recuperado el 21 de septiembre de 2019 de https:/ebevidencia.com/wp-content/uploads/2014/12/analisis-datoscualitativos.pdf [GS Search]

Flores-Camacho, F. (2012). La enseñanza de la ciencia en la educación básica en México. México: INEE. ISBN 978-607-7675-39-6. Recuperado de https://www.inee.edu.mx/wpcontent/uploads/2019/01/P1C227.pdf [GS Search]

Gil, J. (2011). Técnicas e instrumentos para la recogida de información. España: Universidad Nacional de Educación a Distancia. ISBN 978-84-3626-250-6. [GS Search]

Hernández, R., Fernández, C., y Baptista, M. (2010). Metodología de la investigación. México: McGraw Hill. ISBN 978-607-15-0291-9. [GS Search]

Hidalgo, L. (2005). Confiabilidad y Validez en el Contexto de la Investigación y Evaluación Cualitativas. Recuperado el 19 de mayo de 2018 de http://www.ucv.ve/uploads/media/Hidalgo2005.pdf

Katayama, R. (2014). Introducción a la Investigación Cualitativa: Fundamentos, métodos, estrategias y técnicas. Perú: Fondo Editorial de la Universidad Inca Garcilaso de la Vega. ISBN 978-612-4050-75-6.

Martínez, R. (2007). La investigación en la práctica educativa: Guía metodológica de investigación para el diagnóstico y evaluación en los centros docentes. España: Ministerio de Educación y Ciencia. ISBN 978-84-369-4440-2.

Martínez-Salgado, C. (2012). El muestreo en investigación cualitativa. Principios básicos y algunas controversias. Ciência \& Saúde Coletiva, 17(3). 613-619. Recuperado de http://www.scielo.br/pdf/csc/v17n3/v17n3a06.pdf

Massot, I., Dorio, I. y Sabariego, M. (2009). Estrategias de recogida y análisis de la información. En R. Bisquerra (ed.), Metodología de la investigación educativa (pp. 329-366). Universidad de Barcelona. ISBN 978-84-7133-748-1. 
MAXQDA. (2018). MAXQDA The Art of Data Analysis. Recuperado el 11 de febrero de 2019 de https://es.maxqda.com/

Muñoz, H. (2016). Mediaciones tecnológicas: nuevos escenarios de la práctica pedagógica. Praxis \& $\quad$ Saber, 7(13). 199-221. $\quad$ Recuperado de https://revistas.uptc.edu.co/index.php/praxis_saber/article/view/4172 [GS Search]

OCDE. (2016). Programa para la evaluación internacional de alumnos PISA 2015 resultados. Recuperado de https://www.oecd.org/pisa/PISA-2015-Mexico-ESP.pdf

Pérez, M. (2017). La importancia del concepto de intervención educativa, Revista Educarnos, Recuperado de: https://revistaeducarnos.com/la-importancia-del-concepto-de-intervencioneducativa/

SEP. (2017). Aprendizajes Clave para la Educación Integral Plan y programas de estudio para la educación básica. México: Secretaría de Educación Pública. ISBN 978-607-97644-0-1. Recuperado

de http://www.aprendizajesclave.sep.gob.mx/descargables/APRENDIZAJES_CLAVE_PARA LA EDUCACION_INTEGRAL.pdf

Sabariego, M. y Bisquerra, R. (2009). El proceso de investigación (parte 1). En R. Bisquerra (ed.), Metodología de la investigación educativa (pp. 89-125). Universidad de Barcelona. ISBN 978-84-7133-748-1.

Strauss, A., y Corbin, J. (2002). Bases de la investigación cualitativa. Técnicas y procedimientos para desarrollar la teoría fundamentada. Colombia: Universidad de Antioquia. ISBN 958655-623-9.

Tesconi, S. (2015). Crear artefactos para generar conocimiento compartido: el modelo de aprendizaje del movimiento maker como herramienta de formación del profesorado. Comunicación y pedagogía: Nuevas tecnologías y recursos didácticos, (284), 40-47. Recuperado https://www.researchgate.net/publication/283205995 Crear artefactos para generar conoc imiento compartido El modelo de aprendizaje del movimiento maker como herramien ta de formacion del profesorado

Tourón, J. (2016). TPACK: un modelo para los profesores de hoy. Recuperado de https://www.javiertouron.es/tpack-un-modelo-para-los-profesores-de/

Walker, M., y Montero, L. (2004). Principios Generales de educación de adultos, Rev Child Med Fam, 5(2), 65-71. $\quad$ Recuperado de https://www.revistachilenademedicinafamiliar.cl/index.php/sochimef/article/view/154

\section{Apéndice}

\section{A. Encuestas e ítems de las entrevistas abiertas}

El listado de las encuestas e ítems de las entrevistas abiertas relacionados a este estudio se puede acceder desde este enlace:

https://drive.google.com/file/d/1k2jR484CeLo3xljDXiyVk8zOEzTO_PA9/view?usp=sharing 